\title{
Examining architectures of photoanode-photovoltaic tandem cells for solar water splitting
}

\author{
Jeremie Brillet, Maurin Cornuz, Florian Le Formal, Jun-Ho Yum, Michael Grätzel, and Kevin Sivula \\ Institut des sciences et ingénierie chimiques, Ecole Polytechnique Fédérale de Lausanne, \\ CH-1015 Lausanne, Switzerland
}

(Received 14 May 2009; accepted 22 July 2009)

\begin{abstract}
Given the limitations of the materials available for photoelectrochemical water splitting, a multiphoton (tandem) approach is required to convert solar energy into hydrogen efficiently and durably. Here we investigate a promising system consisting of a hematite photoanode in combination with dye-sensitized solar cells with newly developed organic dyes, such as the squaraine dye, which permit new configurations of this tandem system. Three configurations were investigated: two side-by-side dye cells behind a semitransparent hematite photoanode, two semitransparent dye sensitized solar cells (DSCs) in front of the hematite, and a trilevel hematite/DSC/DSC architecture. Based on the current-voltage curves of state-of-the-art devices made in our laboratories, we found the trilevel tandem architecture (hematite/SQ1 dye/N749 dye) produces the highest operating current density and thus the highest expected solar-to-hydrogen efficiency (1.36\% compared with $1.16 \%$ with the standard back DSC case and $0.76 \%$ for the front DSC case). Further investigation into the wavelength-dependent quantum efficiency of each component revealed that in each case photons lost as a result of scattering and reflection reduce the performance from the expected 3.3\% based on the nanostructured hematite photoanodes. We further suggest avenues for the improvement of each configuration from both the DSC and the photoanode parts.
\end{abstract}

\section{INTRODUCTION}

Since the seminal demonstration of photoelectrochemical (PEC) water splitting with $\mathrm{TiO}_{2}{ }^{1}$, scientists and engineers have relentlessly searched for a PEC watersplitting material combining efficient solar light harvesting, high quantum efficiency, practical durability, and low cost. However, no single semiconducting material has yet been found to meet all of these requirements. While systems delivering high efficiency have been demonstrated using III-V semiconductor materials with optimized band gaps and energy levels straddling the hydrogen and oxygen redox potentials, their cost and stability are a major disadvantage. ${ }^{2}$ Oxide semiconductors such as $\mathrm{WO}_{3}$ and $\mathrm{Fe}_{2} \mathrm{O}_{3}$, which have reasonable band gap energies $\left(E_{\mathrm{g}}\right)$ and high stability in aqueous electrolytes, have also been thoroughly investigated as photoanodes for water splitting. ${ }^{3,4}$ The doping and morphology of these materials have been engineered to improve their performance, but the conduction band edge energies in these semiconducting oxides are intrinsically too low to evolve hydrogen, and an external bias must be applied to split water. ${ }^{5}$ To address this shortcoming,

\footnotetext{
a) Address all correspondence to this author.

e-mail: kevin.sivula@epfl.ch

DOI: 10.1557/JMR.2010.0009
}

systems using more than one semiconductor have been proposed. ${ }^{6}$ In addition, since oxide semiconductors such as $\mathrm{WO}_{3}$ and $\mathrm{Fe}_{2} \mathrm{O}_{3}$ do not absorb all of the incoming solar irradiation $\left(E_{\mathrm{g}}=2.6\right.$ and $2.0 \mathrm{eV}$, respectively), if they are deposited on a transparent conducting substrate, a portion of the solar irradiation $\left(h v<E_{\mathrm{g}}\right)$ will not be absorbed. Transmitted photons can then be converted by another photosystem to provide the necessary potential to complete the water-splitting reaction and accomplish unassisted solar hydrogen production from water. This second photosystem is most practically a photovoltaic (PV) device as photocathode materials typically suffer from stability issues. In addition, for two-photon configurations, the upper efficiency limit for a separately illuminated photocathode-photoanode PEC is approximately $10 \%$, while for a tandem PEC system it is more than $18 \%{ }^{6}$

Any reasonable tandem cell system must be potentially constructed with inexpensive materials and processes to be a realistic solution to our global energy demand. Our group has previously focused research efforts on hematite $\left(\alpha-\mathrm{Fe}_{2} \mathrm{O}_{3}\right)$ as the photoanode material. In addition to its chemical stability in aqueous environments and matchless abundance, its band gap of $2.0 \mathrm{eV}$ makes hematite an ideal photoanode material capable of converting up to $16 \%$ of the incident solar energy into 
hydrogen in a tandem cell system. ${ }^{7}$ In addition, our recent efforts to tune the material properties of hematite prepared by a simple atmospheric pressure chemical vapor deposition (APCVD) method has resulted in highly nanostructured photoanodes that deliver unmatched photocurrents under standard solar conditions. ${ }^{8}$ Prepared on $\mathrm{F}: \mathrm{SnO}_{2} /$ glass substrates, these hematite photoanodes transmit solar photons with wavelengths longer than $\sim 600 \mathrm{~nm}$ to a tandem PV cell. A promising candidate for this photovoltaic device is the dye-sensitized solar cell (DSC) as these devices can be produced for onethird to one-fifth the cost of a conventional silicon solar cell $^{9}$ and the absorption range can be tuned by selecting the appropriate dye.

Ideally in this hematite/DSC water-splitting tandem cell, only one photovoltaic system would provide the necessary potential to complete the water-splitting reaction. ${ }^{10}$ However, because of the large overpotential needed for water oxidation and other loss processes, two solar cells are needed to provide enough voltage to the current state-of-the-art $\mathrm{Fe}_{2} \mathrm{O}_{3} .{ }^{8}$ This triple-junction photo-rectifier architecture does not fundamentally provide a limitation to the possible solar-to-hydrogen conversion efficiency, as less than one-third of the available solar photons have wave length shorter than $600 \mathrm{~nm}$ and panchromatic dyes with high quantum efficiency extending past $900 \mathrm{~nm}$ are being developed. ${ }^{11}$ However it does present a challenge to device construction that has been addressed by placing two series-connected DSCs abreast and tandem with the photoanode as shown in Fig. 1(a). ${ }^{12}$ In this way the two DSCs would be constructed each with half of the active area of the photoanode to normalize the total area of the device.

Recently, the development of new dyes for the DSC opens up new possibilities for optimizing light harvesting in these water-splitting tandem cells. All-organic dyes, such as the squaraine dye, have a narrow absorption bandwidth extending into the far red region of the visible spectrum and have demonstrated solar power conversion efficiencies up to $4.5 \%$ under AM 1.5. ${ }^{13} \mathrm{In}$ addition to considerably reducing costs when compared with a ruthenium-based dye, two new distinct tandemcell configurations become available when using this type of dye. First we can propose a trilevel tandem device [Fig. 1(b)]. $\mathrm{A} \mathrm{Fe}_{2} \mathrm{O}_{3} /$ DSC-squaraine dye/DSC-panchromatic dye configuration would eliminate the need to construct two DSCs side-by-side. A second possibility with the squaraine dye is to arrange two-abreast DSCs in front of a hematite photoanode as in Fig. 1(c). This "front DSC" configuration is particularly attractive in view of light harvesting as hematite has a high index of refraction that increases reflection. In addition, the front DSC approach would eliminate the need to deposit the photoanode on a transparent conducting glass and an inexpensive metal foil support could instead be used. To determine how
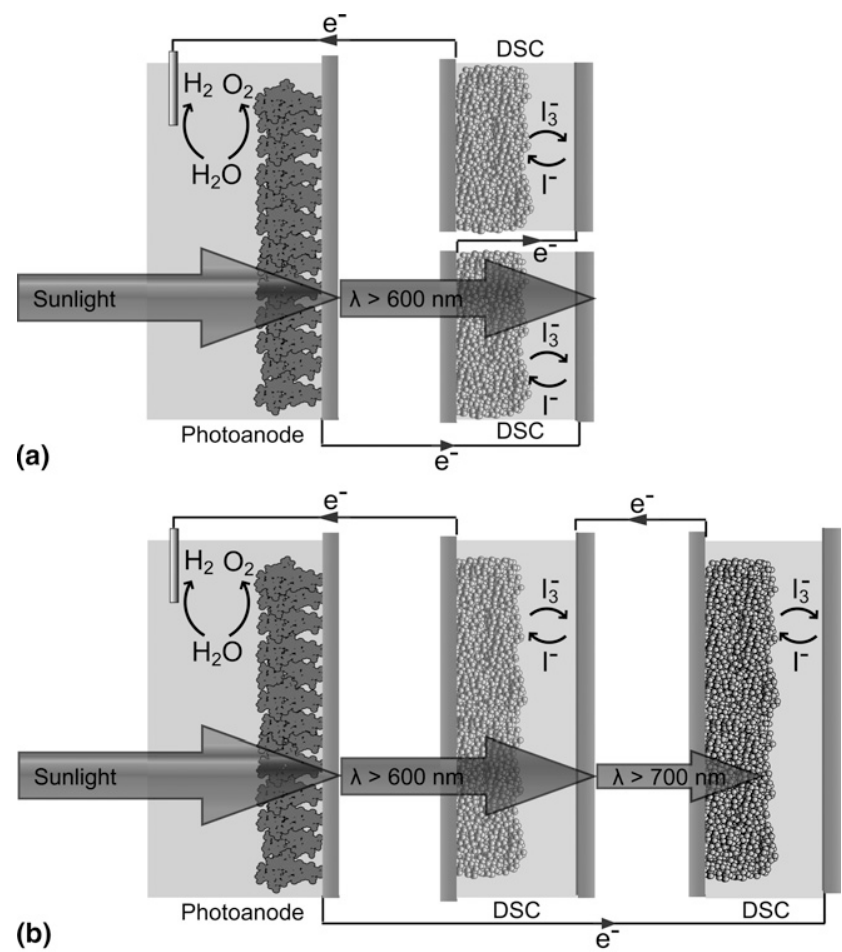

(b)

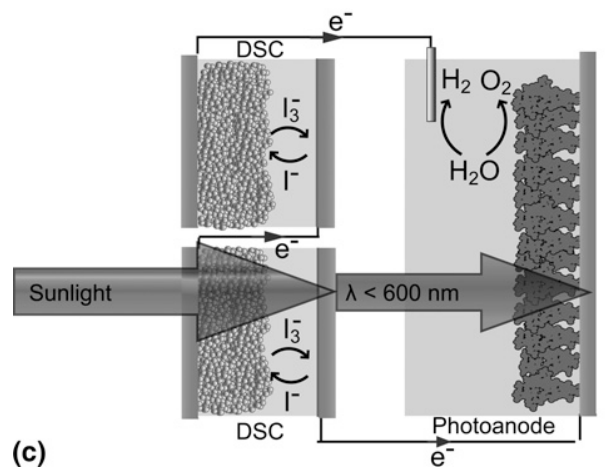

FIG. 1. Layout of three architectures for a tandem cell using a hematite photoanode and two dye-sensitized solar cells in series. (a) "Back DSC" configuration. (b) "Trilevel" configuration. (c) "Front DSC" configuration.

these two new hematite/DSC tandem concepts "stack up," here we present their investigation compared with the so-called standard "back DSC" system achieved by scrutinizing the wavelength-dependant performance after having evaluated the expected STH conversion efficiencies based on the performance of the actual devices made in our labs.

\section{EXPERIMENTAL}

Silicon-doped hematite photoanodes were made by atmospheric pressure chemical vapor deposition (APCVD) from $\mathrm{Fe}(\mathrm{CO})_{5}$ (Aldrich 99.999\%) and tetraethoxylane (TEOS; Aldrich 99.999\%) on $\mathrm{F}: \mathrm{SnO}_{2}$-coated glass (TEC15, Hartford Glass) at $420{ }^{\circ} \mathrm{C}$ as previously reported in the literature. ${ }^{8}$ Their current-voltage response under 
global AM 1.5 simulated solar irradiation $\left(1000 \mathrm{~W} / \mathrm{m}^{2}\right)$ and in the dark were measured together with their incident photon conversion efficiencies (IPCEs) at different potentials in a two-electrode system (with a platinum wire cathode) to account for all overpotentials and resistances. The electrolyte was $1 \mathrm{M} \mathrm{NaOH}$ (Fluka, pro analysis in Milli-Q water, $25^{\circ} \mathrm{C}, \mathrm{pH}=13.6$ ) deoxygenated by bubbling nitrogen.

A detailed methodology of fabricating and characterizing the dye-sensitized solar cell is well described in the literature. The dyes were selected to allow a broad transparency window in the visible spectrum for the first cell and maximizing the absorption range for the second cell. A squaraine dye coded as SQ1 $1^{13}$ and the black dye $(\mathrm{N} 749)^{11}$ were chosen based on their complementary properties over their absorption spectra. The electrolyte A6141 ${ }^{14}$ was selected for its electrochemical properties allowing a high current density with low concentration and therefore high light transmission. The N749 dye and A6141 electrolyte were synthesized in our labs, and the SQ1 dye is the courtesy of Pr. F. Nüesch, EMPA. Transparent $\mathrm{TiO}_{2}$ layers were used $(20 \mathrm{~nm}$ particles, synthesized in house) to avoid light scattering. The thickness of the semiconducting layer in the squaraine-sensitized cell was carefully optimized to balance in each case between an improvement of the transmission properties and the needed photocurrent matching between the series plugged photosystems. The best result is presented in this study. The thickness of the black dye-sensitized cell was selected to match the current of the squaraine cell when the two devices are superimposed. The current-voltage responses of these devices were measured under the simulated AM $1.5 \mathrm{G}\left(1000 \mathrm{~W} / \mathrm{m}^{2}\right)$. The appropriate device (hematite photoanode or/and SQ1 DSC) was used as an incident light filter according to the considered architecture. The incident photon-to-current efficiencies (IPCE) were measured under standard (short-circuit) conditions. The transmittance of each tandem cell component was measured by a Hewlett-Packard 8452A diode array ultraviolet-visible (UV-Vis) spectrophotometer.

\section{RESULTS AND DISCUSSION}

As suggested by Weber and Dignam ${ }^{6}$ the operating water-splitting current density, $J_{\mathrm{op}}$, in a tandem cell is estimated by measuring the current-voltage (IV) curves, separately, of the hematite photoanode and the two DSCs in series with the actual light incident to that separate part given its position in the particular tandem configuration, and then overlapping the IV plots of the DSCs in series and the photoanode. Figure 2 illustrates the concept of finding $J_{\text {op }}$ in the ideal case. The operating potential $U_{\text {op }}$ is defined as the potential that should be measured at a common node between the PV and the PEC cell. Next we calculate the overall direct solar-

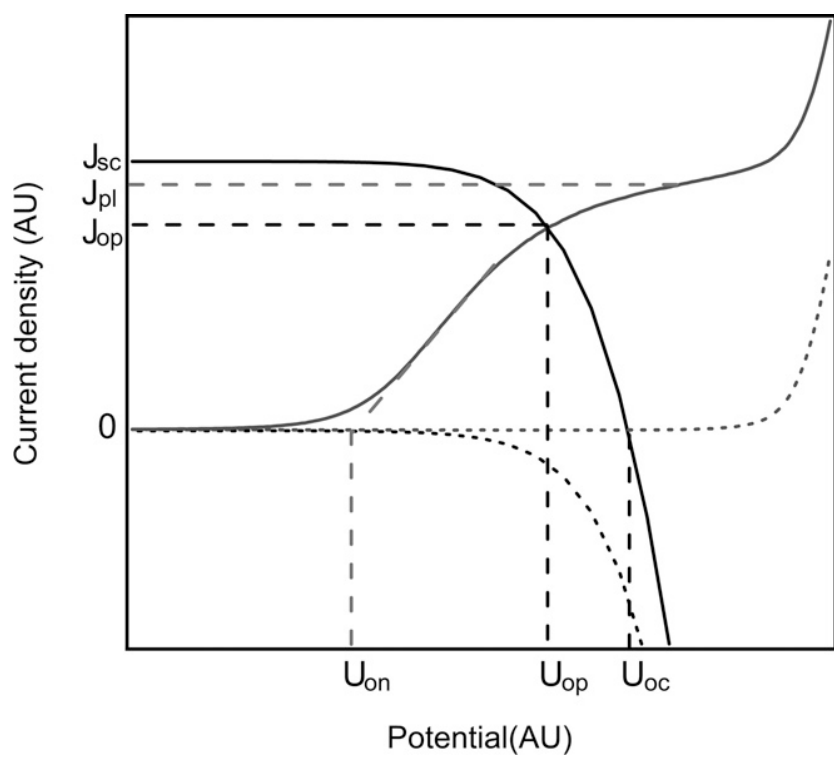

FIG. 2. Current-voltage curves for a dye-sensitized solar cell (black) on top of a hematite photoanode (gray) for ideal case in the dark (broken lines) and in under illumination (solid lines). Relevant parameters of the curves are indicated and described in the text.

to-hydrogen efficiency based on the amount of hydrogen produced, which is given by:

$$
V=\frac{J_{\mathrm{op}} \cdot V_{\mathrm{m}}}{n F},
$$

where $V$ is the volume of hydrogen produced $\left(\mathrm{L} / \mathrm{s} \cdot \mathrm{cm}^{2}\right)$, $J_{\text {op }}$ the operating current density $\left(\mathrm{A} / \mathrm{cm}^{2}\right)$ at the IV curves intersection point, $V_{\mathrm{m}}$ is the molar volume of the gas at STP, $F$ the Faraday constant $(96,485 \mathrm{C} / \mathrm{mol}), n$ the number of electrons involved (two in this case). Here we assume all of the electrons counted in the external circuit would be involved in the water oxidation and reduction reactions; that is, no competing electrochemical reactions are occurring. This is reasonable given the stability of the hematite/base electrolyte system.

While the aforementioned approach gives the expected hydrogen production rate by each tandem cell, it gives little information as to the limitations of each system. To fully understand the optical limitations of each system it is judicious to look at the photon-to-current conversion efficiency of each component separately as a function of incident wave length $[\operatorname{IPCE}(\lambda)]$ over the solar spectrum. Then the total current density delivered by each component can be calculated using:

$$
J_{\mathrm{V}}=e \int \operatorname{IPCE}(\lambda) \Phi_{\text {photon }}(\lambda) d \lambda,
$$

where $J_{\mathrm{V}}$ is the current density at the considered potential, $e$ is the elementary charge, and $\Phi_{\text {photon }}(\lambda)$ the incident photon flux as a function of the wave length. The last term, $\Phi_{\text {photon }}(\lambda)$, is easily calculated for each component by multiplying the measured transmittance of 
any filtering components (in front of the component in question) by the global AM 1.5 solar spectral irradiation. ${ }^{15}$ The IPCE of the hematite photoanode was evaluated at the voltage of the IV curve intersection point potential $\left(U_{\mathrm{op}}\right)$. The IPCE of PV and PEC devices is strongly dependant on the potential at which the photoelectrochemical cell is operating. The IPCE of the DSCs at $U_{\text {op }}$ were therefore estimated by measuring the photocell IPCE under short-circuit conditions. We then supposed the linearity of the DSCs over illumination power along with the uniform scaling of the IPCE $(\lambda)$ at different potentials ${ }^{16}$ and renormalized the IPCE to match the calculated currents, $J_{\mathrm{v}}$, with the current predicted by the overlapping of the IV curves, $J_{\text {op }}$, to account for the difference of the operating potential and the short-circuit measurement conditions. Finally, the IPCEs and transmittances were compared in each case to fully understand the limitations of the studied architecture and propose routes for optimization. Figures 3 to 5 summarize the principle characteristics of each configuration. Additionally, the photoanode and photovoltaic IV parameters along with the operating potential $U_{\text {op }}$, operating current density $J_{\mathrm{op}}$, corresponding dihydrogen evolution capacity $V\left(\mathrm{H}_{2}\right)$, and direct solar-to-hydrogen

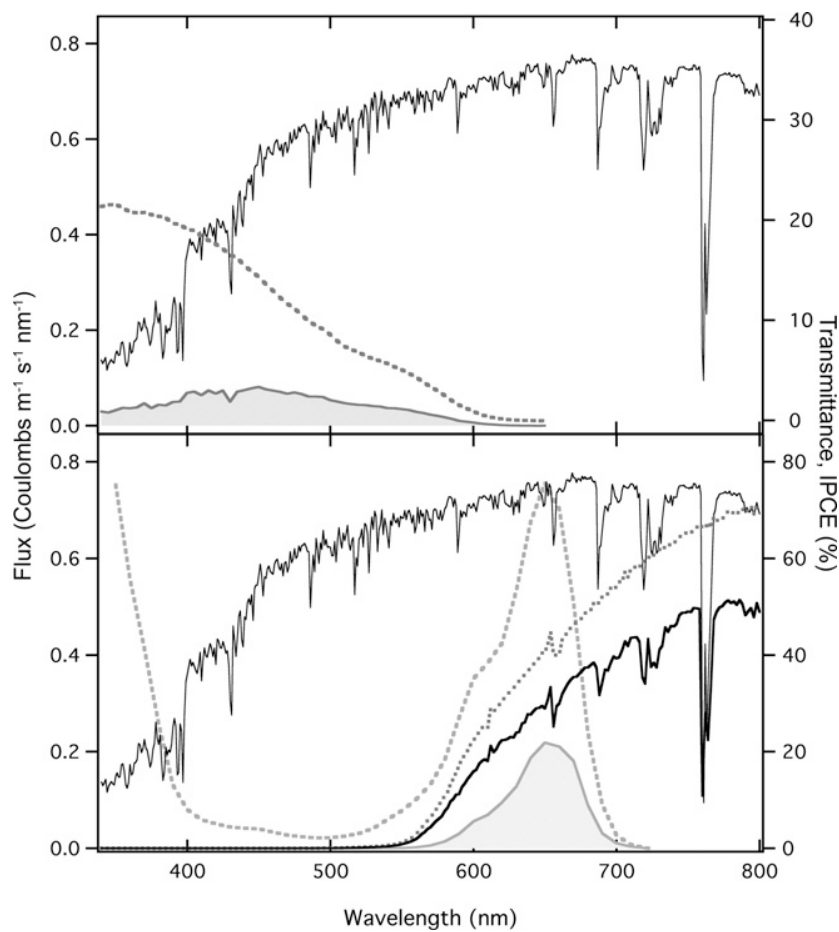

FIG. 3. Optical and electronic characteristics of the "back DSC" tandem cell. Top panel: Solid line shows incident solar flux. The IPCE of the hematite (dashed line) is convoluted with the solar flux to give the electron flux in the photoanode (shaded area). Bottom panel: The solar flux is convoluted with the transmittance of the hematite (dotted line) to give the incident irradiance on the DSC (solid bold line). The irradiance is convoluted with the IPCE of the DSC (dashed line) to give the electron flux in the photovoltaic device (shaded area). conversion efficiency \%STH (calculated based on the upper heating value of $286 \mathrm{~kJ} / \mathrm{mol}$ or $1.45 \mathrm{eV} /$ electron for dihydrogen) for each tandem architecture are summarized in Table I.

For the standard "back-DSC" configuration we observed an operating current density close to the shortcircuit current density of the DSC $\left(J_{\mathrm{op}}, J_{\mathrm{sc}}=0.80\right.$, $0.85 \mathrm{~mA} / \mathrm{cm}^{2}$ ) but far from the plateau current of the photoanode $\left(2.67 \mathrm{~mA} / \mathrm{cm}^{2}\right)$. This is partly because of our choice to use the squaraine dye for this configuration to better compare with the other systems presented. While this dye, which absorbs light between 600 and $800 \mathrm{~nm}$, is nominally compatible with the band gap of hematite $(600 \mathrm{~nm}$, see Fig. 3, top) in practice we observe that less than $50 \%$ of the solar irradiation is transmitted through the photoanode to the DSCs at the IPCE max of $650 \mathrm{~nm}$ as shown in the bottom panel of Fig. 3. This unexpected result is caused by light scattering and reflection from the photoanode and reduces the shortcircuit current of the DSC by $20 \%$ over what is observed under direct solar illumination (see front DSC $J_{\mathrm{sc}}$ ). To investigate improving the light harvesting in DSCs in this case, we determined the operating potential and current using the panchromatic black dye in the DSCs

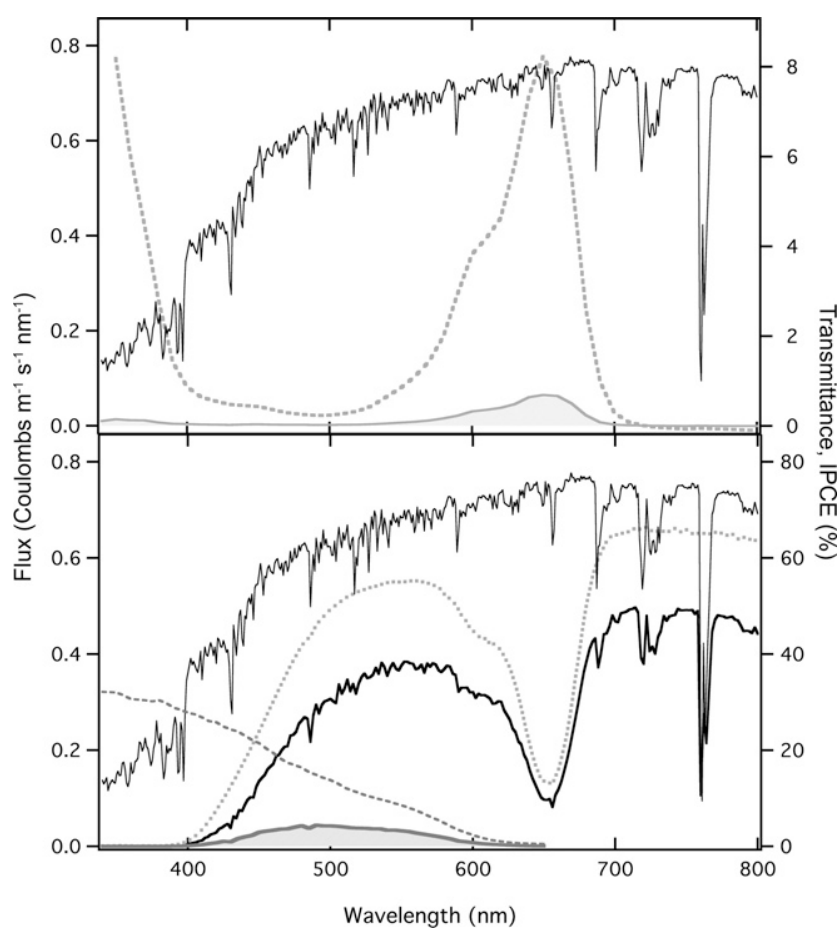

FIG. 4. Optical and electronic characteristics of the "front DSC" tandem cell. Top panel: Solid line shows the incident solar flux. The IPCE of the DSC (dashed line) is convoluted with the solar flux to give the electron flux in the DSC (shaded area). Bottom panel: The solar flux is convoluted with the transmittance of the DSC (dotted line) to give the incident irradiance on the hematite (solid bold line). The irradiance is convoluted with the IPCE of the photoanode (dashed line) to give the electron flux in hematite (shaded area). 


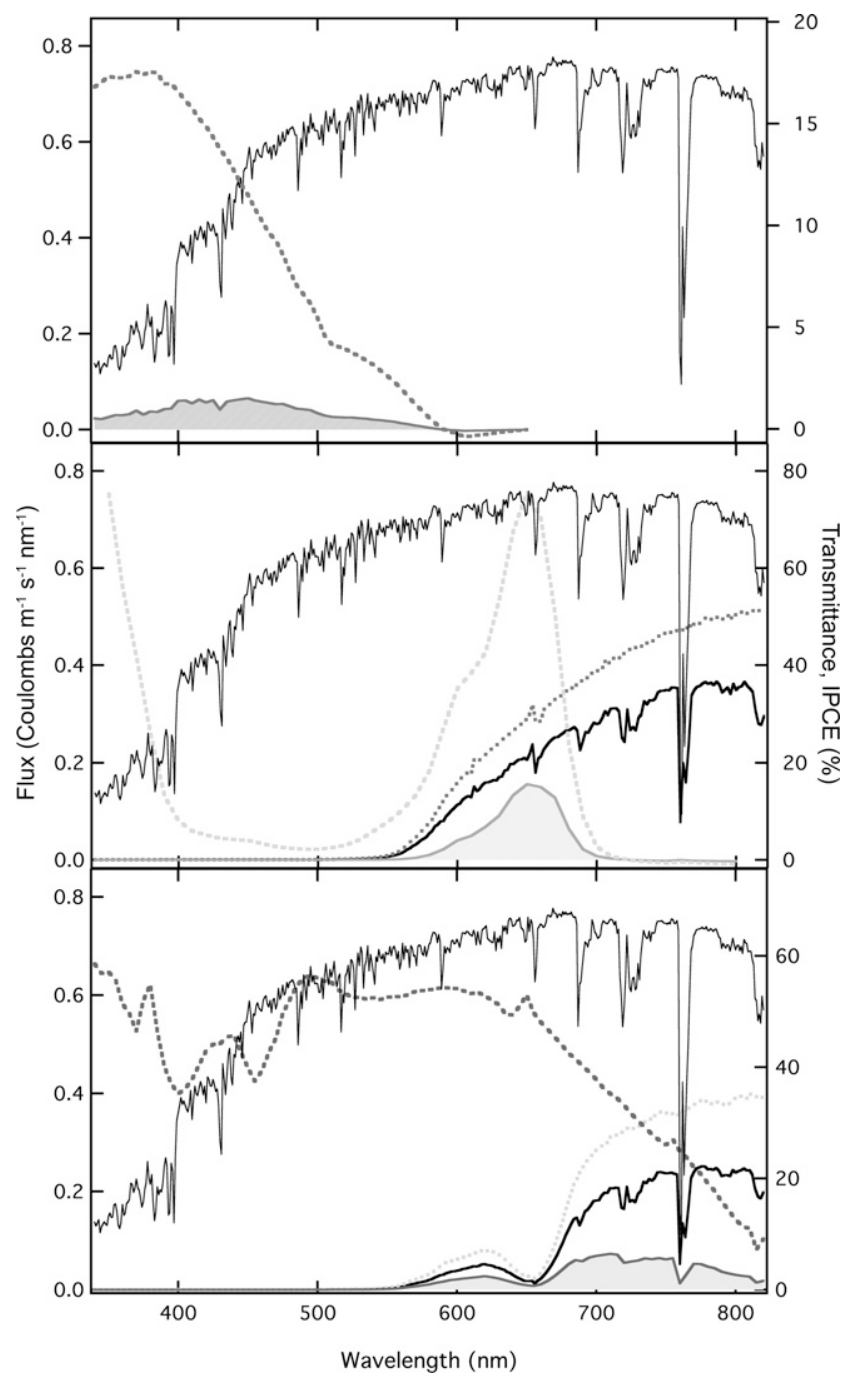

FIG. 5. Optical and electronic characteristics of the "trilevel" tandem cell. Top panel: Solid line shows the incident solar flux. The IPCE of the hematite (dashed line) is convoluted with the solar flux to give the electron flux in the photoanode (shaded area). Middle panel: The solar flux is convoluted with the transmittance of the hematite (dotted line) to give the incident irradiance on the squaraine DSC (solid bold line). The irradiance is convoluted with the IPCE of the squaraine DSC (dashed line) to give the electron flux in the intermediate photovoltaic device (shaded area). Bottom panel: The solar flux is convoluted with the transmittance of the squaraine dye plus hematite (dotted line) to give the incident irradiance on the black DSC (solid bold line). The irradiance is convoluted with the IPCE of the black DSC (dashed line) to give the electron flux in the bottom photovoltaic device (shaded area). instead of the squaraine dye. In this case we found that, because of the light reflection and scattering as well as a deceasing IPCE of the black dye cells in the wavelength range of 650 to $800 \mathrm{~nm}$, the $J_{\mathrm{sc}}$ of the solar cells only improved to $1.03 \mathrm{~mA} / \mathrm{cm}^{2}$. In addition, because of the lower $U_{\text {oc }}$ offered by the black dye cells $(1.32 \mathrm{~V})$ the operating potential and current both were reduced (to $1.22 \mathrm{~V}$ and $0.61 \mathrm{~mA} / \mathrm{cm}^{2}$, respectively) actually lowering the hydrogen production expected in this configuration. As neither the squaraine DSCs nor the black dye DSCs were able to produce a short-circuit current with magnitude close to the plateau current of the photoanode in this case, we can conclude that even using dyes with tuned absorptions in the orange to far red part of the spectra, the light lost by reflection and scattering from the photoanode severely limits the performance of the standard "back DSC" architecture. However the "front DSC" configuration [Fig. 1(c)] can potentially eliminate these lost photons.

When considering this "front DSC" architecture, we attempted to select parameters of cell construction to have the maximum transparency possible. We used thin $\mathrm{TiO}_{2}$ layers made with only $20 \mathrm{~nm}$ particles to reduce scattering and make transparent films, a low concentration volatile electrolyte, and a dye leaving a wide transparent window in the hematite absorption region. Even with these factors, we were still able to construct squaraine dye cells with an impressive IPCE maximum of more than $70 \%$ at short-circuit conditions (not shown). Here we observe the photon-to-current conversion of the dye between 550 and $700 \mathrm{~nm}$. The IPCE plot also shows a contribution from the $\mathrm{TiO}_{2}$ at wavelengths below $400 \mathrm{~nm}$. Since the hematite photoanode has low quantum efficiency between 550 and its band gap of $600 \mathrm{~nm}$, and few solar photons are available at wavelengths below $400 \mathrm{~nm}$, the squaraine dye cell seems ideally suited for the front DSC architecture. Despite this, the optical transmittance of the top component is still a limiting factor in the hydrogen production. The transmittance, presented in the bottom panel of Fig. 4, clearly shows a strong absorption of light in the region where the iron oxide has high efficiency $(400-550 \mathrm{~nm})$. In addition, the short wavelength photon flux (300-400 nm) is completely absorbed by the DSC before hitting the hematite. These photons would be converted to current very efficiently by the

TABLE I. Summary of the performance parameters for each tandem cell architecture.

\begin{tabular}{lcccccc}
\hline \hline Architecture & $J_{\mathrm{sc}}\left(\mathrm{mA} / \mathrm{cm}^{2}\right)$ & $U_{\mathrm{oc}}(\mathrm{mV})$ & $J_{\mathrm{pl}}\left(\mathrm{mA} / \mathrm{cm}^{2}\right)$ & $U_{\mathrm{op}}(\mathrm{mV})$ & $J_{\mathrm{op}}\left(\mathrm{mA} / \mathrm{cm}^{2}\right)$ & $\begin{array}{c}V\left(\mathrm{H}_{2} \text { at STP }\right) \\
\left(\mathrm{mL} / \mathrm{cm}^{2} \cdot \mathrm{h}-{ }^{1}\right)\end{array}$ \\
\hline Back DSC & 0.85 & 1420 & 2.67 & 1240 & 0.8 & 0.33 \\
3 levels & 1.59 & 1350 & 2.67 & 1270 & 0.94 & 0.39 \\
Front DSC & 1.06 & 1440 & 0.75 & 1360 & 0.52 & 0.16 \\
\hline \hline
\end{tabular}

First the measured device properties photovoltaic short circuit current density, $J_{\mathrm{sc}}$ and open circuit voltage $V_{\mathrm{oc}}$ as well as the plateau photocurrent of the photoanode are given. The operating potential $U_{\text {op }}$, operating current $J_{\text {op }}$, corresponding dihydrogen evolution capacity $V\left(\mathrm{H}_{2}\right)$, direct solar to hydrogen conversion efficiency $\%$ STH for each tandem architecture follow. 
photoanode. The poor transmission of the squaraine DSCs is the result of both the absorption of the $\mathrm{TiO}_{2}$ and the tri-iodine/iodide redox couple - the latter having a high extinction coefficient and undesirable absorption that peaks at $362 \mathrm{~nm}$. Both of these factors cause a significant attenuation of the incident radiation to the hematite, reducing its plateau photocurrent to $0.75 \mathrm{~mA} / \mathrm{cm}^{2}$, and despite a higher short-circuit current and open-circuit voltage offered by the DSCs in this case $\left(1.06 \mathrm{~mA} / \mathrm{cm}^{2}\right.$, and $1440 \mathrm{mV}$ ) the operating current of the tandem cell is lower than the "back DSC" case by $35 \%$ resulting in a STH of only $0.76 \%$. These results offer a wide avenue for improvement which, on the dye cell side, could begin with an optimization of the choice and concentration of the redox mediator in the electrolyte to increase the transmission in the 400 to $550 \mathrm{~nm}$ range. Alternately, the volume of electrolyte needed could be reduced by including a porous $\mathrm{SiO}_{2}$ layer (spheres) between the electrodes of the DSC as a spacer. ${ }^{17}$ We are actively pursuing these routes to improve the transparency as well as tuning the dye absorption to further optimize this promising configuration which, in addition to eliminating the use of a transparent substrate for the photoanode, reduces the problems of reflection and scattering by the hematite photoanode.

The two above configurations both use two DSCs arranged side by side to provide enough potential to drive the hematite photoanode. Not only does this potentially complicate the device construction, by requiring a small space between the cells that reduces the active area, but to scale the devices with the same active area the current density passing through each DSC must be twice that of the hematite. Even if the light harvesting was optimized, this would be a limitation for the above architectures, given the quantum efficiencies of the dyes used, if the photoanode performed more efficiently. A trilevel tandem [Fig. 1(b)] would eliminate the current density mismatch, and each component in this inline formation would experience the same current flux under operation. As considering the "trilevel" configuration, we chose the Black dye (N749) for the bottom component given its ability to absorb and convert light in the far-red region. This DSC was optimized to absorb and convert the most light possible and to give the highest voltage, as transmission is not an issue. We also note that the SQ1 dye is well suited for application in the middle cell, and it can absorb and convert photons between that of the hematite and of the black dye. The transparent components we already optimized for the "front DSC" configuration were used here, and they do indeed have absorption spectrum narrow enough to permit light conversion by the bottom cell. Looking at the complete outline of the trilevel system's light harvesting, shown in Fig. 5, we note that the top and middle cell have similarly shaped quantum efficiencies and transmission to the front DSC case, except with a different magnitude IPCE and integrated photon flux given the different operating point. In addition, the previous discussions of the light transmission of both the photoanode and the squaraine DSC are appropriate to describe the amount of photons reaching back cell-only about onethird of the available solar photons in the target region of 700 to $800 \mathrm{~nm}$ reach the back cell. Despite this, our analysis of this system yields the highest performance, with an operating current density, $J_{\mathrm{op}}$, of $0.94 \mathrm{~mA} / \mathrm{cm}^{2}$, and an expected STH of $1.36 \%$. This surprising outcome is the result of the increased current density permitted by the trilevel tandem over the other configurations as discussed previously. Indeed, the short-circuit current density of the solar cells in series (and tandem in this case) is the highest at $1.59 \mathrm{~mA} / \mathrm{cm}^{2}$. Incidentally, we also observe a small contribution to the photocurrent by solar photons not absorbed by the squaraine cell between 550 and $650 \mathrm{~nm}$. Even though this system performs the best, we can consider routes for its improvement- two approaches can be considered. An increase in the IPCE of the black dye above $650 \mathrm{~nm}$ will raise the current in the system. This can be done for instance with the use of photonic crystals that would produce slow photons and enhance the light capture in this wave length region. ${ }^{18}$ To further enhance the performance of the trilevel system with hematite focus must be placed on the dyes. The optimal set of band gaps for light harvesting in a trilevel tandem cell are $2.3 \mathrm{eV}(540 \mathrm{~nm}), 1.4 \mathrm{eV}(890 \mathrm{~nm})$, and $0.8 \mathrm{eV}(1550 \mathrm{~nm}) .{ }^{19}$ While the optimal band gap requirements for this hematite water splitting is convoluted with the necessity to have sufficient voltage drive the photoanodes, it is clear that the development of a farinfrared dye pushing the absorption and conversion past $1000 \mathrm{~nm}$ is necessary. This could then replace the black dye in the bottom cell and a more broadly absorbing and high-voltage dye, like $\mathrm{N} 719,^{20}$ could be used for the middle cell.

In general, our analysis has shown that light scattering, reflection, and unwanted component absorption are major limitations to the hematite/DSC water-splitting tandem cell system. However, since the components of our system are all constructed with similar conductive glass components, the construction of monolithic tandem cells without concern for dissimilarity in thermal expansion is possible and would no-doubt improve the light harvesting. ${ }^{21}$ This, and the continued development of dyes with tuned absorption, especially in the infrared region are necessary to improve the light harvesting. In addition, a photoanode delivering a higher plateau current is necessary for the further development of this system. An increase in the IPCE of the hematite photoanode, especially in the near band gap region, would improve the "front DSC" case remarkably. We are currently working toward optimizing the light-harvesting and quantum efficiency of the hematite photoanodes in our laboratories. 
In addition to the aforementioned limitations, it is important to note that, in all three tandem cell configurations considered, the operating current density was small compared with the plateau current offered by the photoanode. While this discrepancy could be interpreted to be a result of an insufficient voltage provided by the photovoltaic devices, it is more realistically a result of the high overpotential needed for the oxygen evolution at the photoanode. Under flat band conditions the valence band holes in our hematite have an energy approximately $1 \mathrm{~V}$ below the $\mathrm{O}_{2} / \mathrm{H}_{2} \mathrm{O}$ redox couple in $1 \mathrm{M}$ $\mathrm{NaOH}$, and only $0.3 \mathrm{~V}$ should be needed to raise the electrons to reduce water. ${ }^{22}$ However, we do not observe a photocurrent onset until $1 \mathrm{~V}$ in our two electrode hematite/Pt electrolysis cell. Since about $0.1 \mathrm{~V}$ can be attributed to restive loses and the overpotential at the $\mathrm{Pt}$ cathode (based on three electrode measurements) ${ }^{8}$ we can ascribe a $0.6 \mathrm{~V}$ overpotential for the oxygen evolution reaction at the photoanode. This significant energy loss is the most important parameter needing optimization for these water-splitting cells. We have previously investigated reducing the photocurrent onset using a $\mathrm{Co}^{\mathrm{II}}$ catalyst, ${ }^{8}$ and other groups have also recently reported similar techniques. ${ }^{23,24}$ Further understanding of the water oxidation kinetics in this system are needed to further reduce the onset potential, ideally to a value requiring only one PV cell to drive the water splitting. We are currently working toward this goal in our laboratory.

\section{CONCLUSION}

Through our analysis of the three possible tandem cell configurations employing hematite photoanodes and DSCs with dyes tuned to improve light harvesting for each configuration, we found that given the current state-of-the-art devices, a trilevel tandem architecture (hematite/squaraine dye/black dye), produces the highest operating current density and thus the highest expected solar-to-hydrogen efficiency $(1.36 \%$ compared with $1.16 \%$ which is the standard back DSC case). This is far below the expected 3.3\% that should be possible with the nanostructured hematite photoanodes used. Reduced light harvesting caused by scattering and reflection are limiting the efficiencies presented here, and while device engineering should improve the operating current density using the current materials, new dyes capable of high quantum efficiencies in the far-red and infrared part of the spectra are needed. In addition, the improvement of the photocurrent and especially the onset potential of the hematite photoanode are needed to fully realize the potential of the hematite water-splitting tandem cell.

\section{ACKNOWLEDGMENTS}

We thank the Swiss Federal Office of Energy (Project No. 102326, PECHouse) and the Marie Curie Research
Training Networks (Contract No. MRTN-CT-2006032474) for financial support.

\section{REFERENCES}

1. A. Fujishima and K. Honda: Electrochemical photolysis of water at a semiconductor electrode. Nature 238, 37 (1972).

2. O. Khaselev and J.A. Turner: A monolithic photovoltaicphotoelectrochemical device for hydrogen production via water splitting. Science 280, 425 (1998).

3. B.D. Alexander, P.J. Kulesza, L. Rutkowska, R. Solarska, and J. Augustynski: Metal oxide photoanodes for solar hydrogen production. J. Mater. Chem. 18, 2298 (2008).

4. R. van de Krol, Y.Q. Liang, and J. Schoonman: Solar hydrogen production with nanostructured metal oxides. J.Mater. Chem. 18. 2311 (2008).

5. M. Gratzel: Photoelectrochemical cells. Nature 414, 338 (2001).

6. M.F. Weber and M.J. Dignam: Efficiency of splitting water with semiconducting photoelectrodes. J. Electrochem. Soc. 131, 1258 (1984).

7. A.B. Murphy, P.R.F. Barnes, L.K. Randeniya, I.C. Plumb, I.E. Grey, M.D. Horne, and J.A. Glasscock: Efficiency of solar water splitting using semiconductor electrodes. Int. J. Hydrogen Energy 31, 1999 (2006).

8. A. Kay, I. Cesar, and M. Gratzel: New benchmark for water photooxidation by nanostructured alpha- $\mathrm{Fe}_{2} \mathrm{O}_{3}$ films. $\mathrm{J}$. Am. Chem. Soc. 128, 15714 (2006).

9. J. Augustynski, G. Calzaferri, J.C. Courvoisier, and M. Gratzel: Photoelectrochemical hydrogen production: State of the art with special reference to IEA's hydrogen programme, in 11th World Hydrogen Energy Conference (11 WHEC), edited by T.N. Veziroglu, C.J. Winter, J.P. Baselt, and G. Kreysa (Dechema, Stuttgart, Germany, 1996), p. 2379.

10. A. Duret and M. Gratzel: Visible light-induced water oxidation on mesoscopic $\alpha-\mathrm{Fe}_{2} \mathrm{O}_{3}$ films made by ultrasonic spray pyrolysis. J. Phvs. Chem. B 109, 17184 (2005).

11. M.K. Nazeeruddin, P. Pechy, T. Renouard, S.M. Zakeeruddin, R. Humphry-Baker, P. Comte, P. Liska, L. Cevey, E. Costa, V. Shklover, L. Spiccia, G.B. Deacon, C.A. Bignozzi, and M. Gratzel: Engineering of efficient panchromatic sensitizers for nanocrystalline $\mathrm{TiO}_{2}$-based solar cells. J. Am. Chem. Soc. $\mathbf{1 2 3}$, 1613 (2001).

12. H. Arakawa, C. Shiraishi, M. Tatemoto, H. Kishida, D. Usui, A. Suma, A. Takamisawa, and T. Yamaguchi: Solar hydrogen production by tandem cell system composed of metal oxide semiconductor film photoelectrode and dye-sensitized solar cell. Solar Hydrogen and Nanotechnology II 65003, 65003 (2007).

13. J.H. Yum, P. Walter, S. Huber, D. Rentsch, T. Geiger, F. Nuesch, F. De Angelis, M. Gratzel, and M.K. Nazeeruddin: Efficient far red sensitization of nanocrystalline $\mathrm{TiO}_{2}$ films by an unsymmetrical squaraine dye. J. Am. Chem. Soc. 129, 10320 (2007).

14. T. Bessho, E. Yoneda, J-H. Yum, M. Guglielmi, I. Tavernelli, H. Imai, U. Rothlisberger, M.K. Nazeeruddin, and M. Gratzel: New paradigm in molecular engineering of sensitizers for solar cell applications. J. Am. Chem. Soc. 131, 5930 (2009).

15. Standard tables for reference solar spectral irradiances: Direct normal and hemispherical on $37^{\circ}$ tilted surface. G 173-03, in Annual Book of ASTM Standards (ASTM International, West Conshohocken, PA, 2003).

16. L.M. Peter: Dye-sensitized nanocrystalline solar cells. Phys. Chem. Chem. Phys. 9, 2630 (2007).

17. S. Ito, S.M. Zakeeruddin, P. Comte, P. Liska, D.B. Kuang, and M. Gratzel: Bifacial dye-sensitized solar cells based on an ionic liquid electrolyte. Nat. Photonics 2, 693 (2008). 
18. A. Mihi, M.E. Calvo, J.A. Anta, and H. Miguez: Spectral response of opal-based dye-sensitized solar cells. J.Phvs. Chem.C 112, 13 (2008).

19. A. Devos: Detailed balance limit of the efficiency of tandem solar-cells. J. Phvs. D 13, 839 (1980).

20. M.K. Nazeeruddin, R. Humphry-Baker, P. Liska, and M. Gratzel: Investigation of sensitizer adsorption and the influence of protons on current and voltage of a dye-sensitized nanocrystalline $\mathrm{TiO}_{2}$ solar cell. J. Phvs. Chem. B 107, 8981 (2003).

21. M. Durr, A. Bamedi, A. Yasuda, and G. Nelles: Tandem dyesensitized solar cell for improved power conversion efficiencies. Appl. Phvs. Lett. 84, 3397 (2004).
22. I. Cesar, K. Sivula, A. Kay, R. Zboril, and M. Gratzel: Influence of feature size, film thickness, and silicon doping on the performance of nanostructured hematite photoanodes for solar water splitting. J. Phvs. Chem. C 113, 772 (2009).

23. Y-S. Hu, A. Kleiman-Shwarsctein, G.D. Stucky, and E.W. McFarland: Improved photoelectrochemical performance of Ti-doped $\alpha-\mathrm{Fe}_{2} \mathrm{O}_{3}$ thin films by surface modification with fluoride. Chem. Commun. 19, 2652 (2009).

24. D.K. Zhong, J. Sun, H. Inumaru, and D.R. Gamelin: Solar water oxidation by composite catalyst $/ \alpha-\mathrm{Fe}_{2} \mathrm{O}_{3}$ photoanodes. $\underline{J}$. Am. Chem. Soc. 131, 6086 (2009). 